\title{
Technology-Based Fall Risk Assessments for Older Adults in Low-Income Settings: Protocol for a Cross-sectional Study
}

\author{
Ladda Thiamwong ${ }^{1,2}, \mathrm{PhD}$; Jeffrey R Stout ${ }^{2,3}, \mathrm{PhD}$; Joon-Hyuk Park ${ }^{2,4}, \mathrm{PhD} ; \mathrm{Xin} \mathrm{Yan}^{5}, \mathrm{PhD}$ \\ ${ }^{1}$ College of Nursing, University of Central Florida, Orlando, FL, United States \\ ${ }^{2}$ Disability, Aging and Technology Cluster, University of Central Florida, Orlando, FL, United States \\ ${ }^{3}$ School of Kinesiology and Physical Therapy, College of Health Professions and Sciences, University of Central Florida, Orlando, FL, United States \\ ${ }^{4}$ Mechanical and Aerospace Engineering Department, University of Central Florida, Orlando, FL, United States \\ ${ }^{5}$ Department of Statistics and Data Science, University of Central Florida, Orlando, FL, United States
}

\author{
Corresponding Author: \\ Ladda Thiamwong, PhD \\ College of Nursing \\ University of Central Florida \\ 12201 Research Parkway \\ Orlando, FL, 32826 \\ United States \\ Phone: 14072830470 \\ Email: 1adda.thiamwong@ucf.edu
}

\section{Abstract}

Background: One-third of older adults have maladaptive fall risk appraisal (FRA), a condition in which there is a discrepancy between the level of fear of falling (FOF) and physiological fall risk (balance performance). Older adults who overestimate their physiological fall risk and report a high FOF are less likely to participate in physical activity. Limited data suggest that the association among FOF, body composition, and physical activity intensity differs by fear severity.

Objective: This study aims to examine the associations among FRA, body composition, and physical activity using assistive health technology, including the BTrackS balance system, bioelectrical impedance analysis, and activity monitoring devices. This study also aims to examine the feasibility of recruitment and acceptability of technologies and procedures for use among older adults in low-income settings.

Methods: This cross-sectional study will be conducted in older adults' homes or apartments in low-income settings in Central Florida, United States. Following consent, participants will be contacted, and our team will visit them twice. The first visit includes questionnaire completion (eg, sociodemographic or FOF) and balance performance test using the BTrackS balance system. The participants will be stratified by the FRA matrix. In addition, they will perform hand grip strength and dynamic balance performance tests. Participants will then be asked to wear the ActiGraph GT9X Link wireless activity monitor on the nondominant wrist for 7 consecutive days. The second visit includes body composition testing and a structured interview about the acceptability of the technologies and procedures.

Results: Ethical approval was obtained from the institutional review board of the University of Central Florida (protocol number 2189; September 10,2020). As of December 2020, participation enrollment is ongoing and the results are expected to be published in Summer 2022.

Conclusions: Accurate FRA is essential for implementing physical activity programs, especially in older adults with low income. This study will provide data for developing technology-based fall risk assessments to improve participation in physical activity, thus enhancing healthy longevity among older adults in low-income settings.

International Registered Report Identifier (IRRID): PRR1-10.2196/27381

(JMIR Res Protoc 2021;10(4):e27381) doi: $\underline{10.2196 / 27381}$

\section{KEYWORDS}

body composition; falls; risk assessment; technology; wearable devices; accidental falls; fear 


\section{Introduction}

\section{Background}

More than 15 million (30\%) older adults in the United States have income below $200 \%$ of the poverty line [1,2]. The poverty rate increases with age and is higher among women, Black and Hispanic individuals, and individuals with poor health [1]. Older adults who live in low-income communities are less likely to engage in physical activity (PA) [3-5], defined as any bodily movement produced by skeletal muscles that results in energy expenditure [6]. Lack of PA is related to chronic conditions and reduced quality of life among older adults with low income $[7,8]$. Limited data suggest that older adults who overestimate their fall risk and report fear of falling (FOF) are less likely to participate in PA, and the association between FOF and PA intensity differs by fear severity [9]. Changes in body composition also have a significant effect on physical functions and quality of life [10]. However, these studies did not include older adults from low-income settings, who were more likely to report falls in the previous year [11].

One-third of older adults have maladaptive fall risk appraisal (FRA), a condition in which there is a discrepancy between the perceived fall risk (levels of FOF) and physiological fall risk (balance performance) [12,13]. Measuring FRA in older adults can be challenging because of self-report bias and cognitive deficit $[14,15]$. Using both subjective and objective measures provides better fall risk assessment among older adults [15,16]. Thus, we developed an FRA matrix, a graphical grid categorizing the levels of FOF and balance performance into 4 quadrants: (1) rational FRA (low FOF and normal balance), (2) incongruent FRA (low FOF despite poor balance), (3) irrational FRA (high FOF despite normal balance), and (4) congruent FRA (high FOF and poor balance) [12]. In our pilot study $(\mathrm{n}=102)$, we measured FOF using a questionnaire and balance performance using a portable and novel force balance plate (BTrackS Assess Balance System [BBS], Balance Tracking System, Inc). We found that $40 \%$ of older adults had maladaptive FRA. In this group, $19 \%$ of patients with incongruent FRA and 30\% with irrational FRA reported falling in the past year [12]. Older adults with congruent FRA or high fear-high physiological were 2.14 times more likely to fall than those with rational FRA or low fear-low physiological [17]. However, our sample included only $11 \%$ of older adults with low income.

\section{Objectives}

Body composition (eg, obesity or low relative skeletal muscle mass) and decline in muscle strength have been associated with FOF, functional impairment, and disability in older American adults [18-20]. Higher BMI and percentage of body fat were associated with poor physical function, whereas the percentage of appendicular lean mass was associated with better physical function (eg, walking or balance test) [19]. Older adults with higher daily PA have better physical function than those who engage in less PA [21]. Although BMI is the most widely used measurement to classify overweight and obesity statuses, it is prone to measurement error and does not consider body fat distribution and skeletal muscle mass [22]. We will use the bioelectrical impedance analysis (BIA) device, which measures body composition and has established normative data among older adults [23].

Research has not examined the associations among body composition, FRA, and PA using assistive health technology (AHT), which applies organizational knowledge, skills, procedures, and systems to improve functioning [24]. This study examines the associations among body composition, FRA, and PA using AHT, including BIA [25], BBS [26,27], and activity monitoring devices [28]. It also aims to examine the feasibility of recruitment and acceptability of technologies and procedures for use among older adults in low-income settings.

\section{Methods}

\section{Study Design}

This is a cross-sectional study.

\section{Settings}

This study will be conducted in older adults' homes or apartments in low-income settings in Central Florida, United States. Collectively, approximately 300 older adults are available for screening at the planned sites.

\section{Study Participants}

We will enroll a sample of 120 participants, if they meet all of the following inclusion criteria: (1) aged $\geq 60$ years, (2) have a low income (using poverty thresholds for 2019 by family size and the number of children aged $<18$ years, published by the US Census Bureau) [29], (3) have no marked cognitive impairment (memory impairment screen score $\geq 5$ [30-32]), and (4) live in their own homes or apartments. Exclusion criteria were as follows: (1) a medical condition precluding balance test (eg, inability to stand on the balance plate) or PA (eg, shortness of breath or feeling pressure when performing PA), (2) currently receiving treatment from a rehabilitation facility, or (3) having medical implants (eg, pacemakers).

\section{Consideration of Sex and Other Relevant Biological Variables}

In our pilot study, $66 \%$ of the participants were women (mean 77 , SD 7.6 years). We found that men tend to cluster in rational and incongruent FRA groups, whereas women were widely distributed among 4 groups (rational, incongruent, irrational, and congruent). We expect that $65 \%$ to $70 \%$ of our sample will be women. We will explore the differences between men and women and between age groups ( $<75$ years vs $>75$ years) to assess whether sex and age as biological variables contribute to the findings.

\section{Variables and Instruments}

The study instruments consist of objective and subjective measures (Table 1). 
Table 1. Description of study variables and instruments.

\begin{tabular}{|c|c|c|}
\hline Variable and instrument & & Description \\
\hline \multicolumn{3}{|l|}{ Participant's characteristics } \\
\hline Sociodemographic and medical history & Self-report questionnaire & $\begin{array}{l}\text { For example, age, gender, living status, education level, socioeconomic } \\
\text { status, perceived general health, comorbidities, mobility problems, medi- } \\
\text { cation use, and number of falls in the past year }\end{array}$ \\
\hline Depressive symptoms & $\begin{array}{l}\text { Patient Health Question- } \\
\text { naire-9 }[33,34]\end{array}$ & $\begin{array}{l}\text { A total of } 10 \text { items (eg, feeling tired) on a 4-point scale; measures symp- } \\
\text { toms of depression within the past } 2 \text { weeks; total scores range } 0-27 \text {; and } \\
\text { scores } \geq 10 \text { indicate moderate depression [33]. Cronbach } \alpha=.89 \text { among } \\
\text { older adults }[33,34]\end{array}$ \\
\hline Cognition & $\operatorname{MIS}^{\mathrm{a}}[30-32]$ & $\begin{array}{l}\text { The MIS is a widely used test of cognitive function and screening } \\
\text { Alzheimer disease among older adults. It is a } 4 \text { - minute, } 4 \text { - item delayed } \\
\text { free and cued recall memory test with controlled learning and high discrim- } \\
\text { inative validity. The maximum score for the MIS is } 8 \text {, and a score of } 5-8 \\
\text { indicates no cognitive impairment and score } \leq 4 \text { indicates possible cognitive } \\
\text { impairment }\end{array}$ \\
\hline
\end{tabular}

$\mathrm{FOF}^{\mathrm{b}}$

Short FES-I ${ }^{\mathrm{c}}$ [35]

Balance performance

Balance performance

Hand grip strength

Body composition

Physical activity

Acceptability of technology
BTrackS balance system $[26,27]$

Sit-to-stand test $[40,41]$

A hand grip dynamometer (JAMAR 5030J1)

Bioelectrical impedance analysis: InBodys 10 device [25]

ActiGraph GT9X Link wireless activity monitors (ActiGraph LLC) [28]

Evaluation form of acceptability of technology and procedures
A total of 7 items (eg, going in or out of a chair) on a 4-point scale; measures concerns about the possibility of falling when performing 7 activities (eg, getting dressed) [36]. Total scores range 7-28 [35]. Higher total scores=higher FOF [37]. Scores 7-10=low concern about falling; scores 11-28=high concern about falling [36,38]. The short FES-I has been validated in community-dwelling older adults [36]. Cronbach $\alpha=.97$ and ICC $^{\mathrm{d}}=0.979$ among older adults [39]

This test consists of 4, 20-second trials. For each trial, the participants will stand as still as possible on the BTrackS Balance Plate with hands on their hips and eyes closed

A sit-to-stand test has been widely used to assess balance and predict falls and was reliable and validated in community-dwelling older adults [40,41]

Hand grip strength will be measured in kilograms as maximal isometric force achieved on a hand grip dynamometer $\left(\mathrm{ICC}=0.959 ; \mathrm{SEM}^{\mathrm{e}}=3.1 \mathrm{~kg}\right.$ )

Participants will remove socks, shoes, and metal objects before the body composition testing. The touch-type electrodes will be placed to the left and right ankles, middle fingers, and thumbs. Participants will hold the position for 1 minute and then remove the touch-type electrodes

Participants will be asked to wear the activity monitoring device on the nondominant wrist for 7 consecutive days. They will be instructed to remove the monitor for imaging studies

Older adults' evaluation of the devices, procedures, and instruments; recommendations for inclusion of older adults in future research

${ }^{\mathrm{a}}$ MIS: memory impairment screen.

${ }^{b}$ FOF: fear of falling.

${ }^{\mathrm{c}}$ FES-I: Fall Efficacy Scale International.

${ }^{\mathrm{d}}$ ICC: intraclass correlation coefficient.

${ }^{\text {e}}$ SEM: standard error of measurement.

Balance performance will be assessed using the BBS [26,27]. The BBS includes a portable BTrackS balance plate (BBP) and BTrackS Assess Balance software running on a computer device. The BBP dimensions are $15.5 \times 23.5 \times 2.5 \mathrm{in}$, weight $6.58 \mathrm{~kg}$, and is operated on Windows 7 or higher via a USB port (Food and Drug Administration approved). During the test, a piece of sturdy furniture or a standard walker will be placed within the participant's reach to reduce the risk of FOF contaminating the performance and to enable even frail people to participate [42]. The software uses the BBS normative database to compare the individual with others in the same age group. The BBS score is dependent on age and sex, but not body size, so that the

percentile rankings can be determined across various age groups and for men and women separately [43]. A scale from 0 to 100 represents the percentile ranking of the BBS. A score of 0 to 30 indicates low fall risk (normal balance) and $\geq 31$ indicates moderate-to-high fall risk (poor balance) [43]. The BBS has excellent validity using Pearson correlations $(r>0.90)$ and high test-retest reliability (intraclass correlation coefficient [ICC $]=0.83$ ) [44].

Body composition will be assessed using a direct segmental multifrequency BIA using the InBodys 10 device [25]. The BIA is manufactured by Biospace Corporation Limited. The BIA 
InBody S10 measures impedance at 6 different frequencies (1, $5,50,250,500$, and $1000 \mathrm{kHz}$ ) for each body segment (right arm, left arm, trunk, right leg, and left leg). The spectrum of electrical frequencies is used in the manufacturer's equation to estimate fat mass, muscle mass, and body water levels. There are no risks, no dunking, no pinching, and no discomfort associated with the use of BIA. The test duration is 1 to 2 minutes. The reliability of the BIA test-retest was high, with an ICC of 0.89 [45].

PA will be measured using activity monitoring devices. All older adults will wear ActiGraph GT9X Link wireless activity monitor (ActiGraph LLC), a triaxial accelerometer, on the nondominant wrist for 7 consecutive days. The GT9X Link has a sample rate of 30 to $100 \mathrm{~Hz}$, a dynamic range of $\pm 8 \mathrm{G}, 14$-day battery life (rechargeable), 180 days/4 GB data storage, and water resistance. Data are collected at 1-minute intervals. A sensor determines whether the device is on or off the wrist. The GT9X Link provides objective 24-hour PA measures, including steps, energy expenditure, activity intensity, and participants' posture. Accelerometry is a reliable method for assessing free-living PA ( $\mathrm{ICC}=0.98$ ) [46] and has been validated against direct observation, energy expenditure, and sedentary behavior $[47,48]$. ActiGraph accelerometers have been used for data collection in the National Health and Nutrition Examination surveys and are the most commonly used devices in research studies [28]. The device display screen can be disabled so that the device does not display the participant's activity but only shows the date and time.

\section{Recruitment Methods}

\section{Identification}

We have created successful, continuing relationships with older adults and staff at the planned sites and demonstrated our ability to recruit a diverse sample [49]. Staff from our community partners and clinical sites will introduce the researchers to older adults to perform the initial screening and determine study eligibility using a checklist.

\section{Recruitment}

Flyers will be posted at our sites, and we will participate in community activities for face-to-face recruitment. Older adults will also be recruited from their homes by local newsletters and word of mouth. The participants will complete an informed consent process before data collection. The research team will maintain a log that tracks screening, eligibility, contact, and recruitment.

\section{Study Procedures and Data Collection}

\section{Training Staff}

All study personnel, including research assistants, will complete the required Collaborative Institutional Training Initiative training for the protection of human subjects. Training will be conducted by the authors (LT, JS, and JP), focusing on research with older adults in low-income settings, communication skills, setting up home visits, study protocols, completing questionnaires, performing tests, and contacting health care providers for older adults identified with a high level of depressive symptoms. We have research assistants who are bilingual and interested in research activities with Hispanic individuals. The authors (LT and JS) will train research assistants to perform balance and body composition tests. The third author (JP) will train research assistants to use accelerometer-based PA devices.

\section{Data Collection}

Following consent, participants will be contacted, and our team will meet with them to complete the questionnaires (eg, sociodemographic, FOF, or Patient Health Questionnaire-9) and the BBS balance performance test. Older adults will take off their shoes and stand as still as possible on the balance plate with their hands on their hips and eyes closed for 2 to 3 minutes. The participants will be stratified by the FRA matrix [12]. We will continually evaluate across the following 4 groups (rational, incongruent, irrational, and congruent) to maintain equal cell distribution (30 older adults per group):

- Rational FRA: low FOF (Short Fall Efficacy Scale International [FES-I] score $\leq 10$ ) and aligned with normal balance (BBS score $=0-30$ ).

- Incongruent FRA: low FOF (Short FES-I score $\leq 10$ ) despite poor balance (BBS score=31-100).

- Irrational FRA: high FOF (Short FES-I score >10) and normal balance (BBS score=0-30).

- Congruent FRA: high FOF (Short FES-I score >10) and poor balance (BBS score $=31-100)$ [12].

In addition, participants will perform hand grip strength and dynamic balance performance tests. Hand grip strength will be measured in kilograms as the maximal isometric force achieved on a hand grip dynamometer. This test will be administered to participants sitting in a chair with their feet flat on the floor and their elbow bent at $90^{\circ}$. The dynamometer will be placed in the hand and adjusted so that the palm side of the grip will be at the palm and the front end will be lined up between the joints of the medial and distal phalanges. The grip size will be adjusted so that the second metacarpals are flat with a $90^{\circ}$ bend at the knuckles. Participants will be asked to squeeze the strength gauge as hard as possible for 3 to 5 seconds. A total of 3 trials on each hand will be performed with a 30 -second rest given between trials. Participants will complete the sit-to-stand test by standing up from a chair as much as possible within 30 seconds [50].

Participants will then be asked to wear the ActiGraph GT9X Link wireless activity monitor on the nondominant wrist for 7 consecutive days. Each participant will be asked to continue their normal activities while wearing the device. The participants will also be instructed and prepared for the body composition test during the second visit. They will be instructed to avoid exercising for 6 to 12 hours, eating for 3 to 4 hours, drinking alcohol or coffee for 24 hours, using shower or sauna, and using lotion or ointment on their hands or feet before the test. Written instructions will be provided to the participants for the use of ActiGraph and body composition tests. The questionnaires and instructions will be provided in English or Spanish by bilingual research assistants who will be trained and randomly monitored to ensure quality and consistency in administering the questionnaires and tests. The participants will be provided with 
a phone number of the study team if they have questions about using the activity monitoring device.

After 7 days of wearing ActiGraph, the research assistants will collect the ActiGraph GT9X Link device. Body composition testing will occur late in the morning, and all participants will be asked to empty their bladder, remove socks, shoes, and metal objects (eg, watches and jewelry) before testing. To ensure that the body is close to a resting state as possible, participants will lie down for 15 minutes before testing in the supine position. If participants select to be tested in a seated or standing position, they will be asked to sit or stand for 10 to 15 minutes before testing (based on participants' ability and preference). Finally, participants will be asked (using our evaluation form) about their reactions to the questions and technology (ie, what they thought about the questionnaires and technology) and asked to describe any concerns or problems in wearing the device (eg, uncomfortable to wear during walking). We will provide the BBS and BIA test results, and participants will receive Can \$37.43 (US \$30) store gift cards upon completion of the study.

\section{Participant Retention}

The following strategies will be used to maximize the participants' retention: (1) obtaining backup contact information (eg, cell phone number or email address); (2) obtaining name and contact information for a person familiar with the participant (eg, family member or neighbor); (3) making confirmation phone calls 2 days before the second visit; (4) assigning the same data collector whenever possible; and (5) making scripted check-in calls, as needed.

\section{Data Management and Analysis}

\section{Data Management and Integrity}

The research assistants will enter deidentified questionnaire data into the Qualtrics Research Suite, a web-based survey tool that allows users to build and analyze web-based surveys and export data in multiple formats, including SPSS. Confidentiality will be maintained using coded data materials. Study data will be maintained on a dedicated server with password-protected access for authorized study staff and encrypted files. The research assistants will assist with data cleaning, preliminary analysis, and data preparation under the supervision of the first and fourth authors (LT and XY). We anticipate no more than $3 \%$ missing values on any one item, as data will be collected in-person on-site at 2 timepoints. The BIA device is supported by a custom software application, which connects to a virtual web-based data platform via a PC. The second author (JS) will conduct these analyses in collaboration with the first and fourth authors (LT and XY).

The ActiLife, the ActiGraph's premier Actigraphy data analysis software platform, will be used to prepare the ActiGraph GT9X Link activity monitors for PA data collection and to download, score, and securely manage the collected data. ActiLife contains a wide selection of algorithms, including steps; energy expenditure; activity bouts such as standing, sitting, or lying down; sedentary analysis; and whether the ActiGraph device has been removed. A bout of sedentary behavior will be defined as consecutive minutes during which the ActiGraph registers less than 100 counts per minute. A break in sedentary behavior will be defined as at least 1 minute in which the counts registered are at least 100 , following a sedentary bout. Activity counts and step data will be used to derive a series of PA and sedentary behavior indicator variables. We will assess the feasibility of wearing the devices by assessing the frequency of older adults who have at least 4 days of at least 10 hours of wear per day (detected using device technology), which is the standard convention [51]. The third author (JP) will conduct these analyses in collaboration with the first and fourth authors (LT and XY).

\section{Data Analysis}

All analyses will be performed in SPSS version 25 or SAS version 9.4, with sufficient annotation for reproducibility. Descriptive and exploratory analyses will be performed first to investigate the distributional assumptions. Although no differences are hypothesized, descriptive subgroup analyses by sex will be conducted.

\section{Aim 1: Examine the Feasibility of Recruitment and Acceptability of Technologies and Procedures for Use Among Older Adults in Low-Income Settings}

We will assess the ability to recruit the sample by calculating the proportions of older adults with low income (1) who are recruited out of the total screened and (2) who completed all study procedures. We will track the number of days and the time spent to recruit the sample. The results will inform the planning for future larger studies. The acceptability of the technologies and procedures will be examined based on an evaluation form (eg, what they thought about the questionnaires and technology) and their recommendations. We will identify the code, categorize participants' responses, and determine the frequency for each category. Demographic data, including essential characteristics of older adults, will be obtained from the study participants. We anticipate no more than $3 \%$ missing values for any one item, as data will be collected in-person on-site.

\section{Aim 2: Examine the Associations Among FRA, Body Composition, and PA}

The hypotheses of the second aim are as follows: (1) rational FRA is associated with higher levels of PA and skeletal muscle mass and with lower levels of percentage of body fat and BMI, (2) incongruent FRA is associated with higher levels of PA and skeletal muscle mass and with lower levels of percentage of body fat and BMI, and (3) irrational and congruent FRAs are associated with lower levels of PA and skeletal muscle mass and with higher levels of percentage of body fat and BMI.

General summary statistics will be presented for continuous data, and percentages will be presented for categorical data. The participants' demographic data will be summarized. Analyses will be performed without adjusting for any covariates, followed by analyses adjusted for age, comorbidities, depressive symptoms, and fall history. The number of comorbidities and the number of falls in the past year will be selected as covariates in the selected model because of their association with FRA, body composition, and PA. Data from InBodys10 (eg, BMI) and ActiGraph (eg, steps per day) could be modeled as continuous. 
Regression models or one-way ANOVA (analysis of variance) will be used to examine the differences in continuous variables (eg, BMI and steps per day) across the 4 groups (rational, incongruent, irrational, and congruent FRAs), and categorical variables (eg, comorbidities) will be tested using chi-square tests. The rational FRA group will set as a base group, and the other 3 groups (incongruent, irrational, and congruent FRAs) will be compared with this base group. Whenever feasible, models will be adjusted for age, sex, day order, wear time (min per day), and fall history. The following variables will be added one by one to evaluate the role of mobility problems (yes or no), depression (score or category), fall risk (high or low), perceived general health (score of category), medication use (yes or no), and comorbidities (yes or no). A final model will include all significant potential variables to evaluate the associations among FRA, body composition, and PA, whenever feasible.

\section{Sample Size and Power Analysis}

There is no historical precedent, and there is limited information to inform the sample size for this study; therefore, the sample size estimate was based on the variable of the BBS score in our pilot study. The mean BBS scores in our pilot study were 26.44 (SD 7.91) in the high PA group and 30.62 (SD 7.78) in the low PA group (PA by self-report), and assuming a two-tail $\alpha$ of .05 and a power of 0.8 , the estimated total sample size of 120 would allow sufficient detection of a mean difference of about 5 hypothetical units of BBS score. Furthermore, to compare the effect sizes of the different body compositions and PA measures, we performed posthoc analyses to compare the mean values between the 2 groups of PA, adjusted for the age group.

\section{Potential Challenges and Solutions}

\section{Recruitment and Retention}

Although recruitment of diverse older adults to research studies is often challenging, our tailored strategies will address this issue by ensuring that our diverse research assistants are well trained and by employing a straightforward data collection process.

\section{Respondent Burden}

We will use questionnaires in either the English or Spanish version, based on the preferences of the older adults. Research assistants will read the questions and help older adults complete if they cannot do so.

\section{Risk of Falls During Tests}

We will follow the test protocol, which was used successfully in our pilot study; we had no falls when using the BBS.

\section{Ethical Approval and Consent to Participate}

Ethical approval was obtained from the institutional review board of the University of Central Florida (protocol number 2189; September 10, 2020). All participants will be informed before their participation.

\section{Results}

The project funding was received on September 30, 2020, and was approved by the Institutional Review Board (September 10, 2020). As of March 2021, we have enrolled 11 participants and expect the results to be published in Summer 2022.

\section{Discussion}

Accurate FRA is essential for implementing PA programs, especially for older adults with low income. Systematic reviews found that most studies have not used objective measures of fall risk and PA [5,52]. Most studies have used self-reported measures of fall risk and PA among older adults [53-57]. Older adults who overestimate their fall risk are less likely to participate in PA [58,59]. Using AHT may eliminate recall bias associated with subjective measures $[60,61]$. Systematic reviews also indicate the importance of technology-based assessments for PA and to prevent falls $[62,63]$. This study will provide data for developing technology-based fall risk assessments to improve participation in PA, thus enhancing healthy longevity among older adults in low-income settings.

\section{Acknowledgments}

This project received financial support from the National Institute on Aging, National Institutes of Health, grant number 1R03AG06799-0, with the projected title, "Technology-based fall risk assessments for older adults in low-income settings." This project is also supported by the Learning Institute for Elders at the University of Central Florida. The funding body is not involved in the study design, data collection, data analysis, or writing of the manuscript.

\section{Authors' Contributions}

LT, JS, JP, and XY contributed to the study conceptualization, design, and data analysis. LT wrote the original draft, and JS, JP, and XY contributed to the substantial revision of the original draft. All authors have agreed to the final version of the paper.

\section{Conflicts of Interest}

None declared.

\section{References}

1. Cubanski J, Koma W, Damico A, Neuman T. How many seniors live in poverty? Kaiser Family Foundation analysis of current population survery, 2018 annual social and economic supplement. 2018. URL: https://www.kff.org/medicare/ issue-brief/how-many-seniors-live-in-poverty/ [accessed 2019-12-12] 
2. The Supplemental Poverty Measure: 2018. United States Census Bureau. URL: https://www.census.gov/library/publications/ 2019/demo/p60-268.html [accessed 2018-12-12]

3. Plow MA, Allen SM, Resnik L. Correlates of physical activity among low-income older adults. J Appl Gerontol 2010 Jul 08;30(5):629-642. [doi: 10.1177/0733464810375685]

4. NCOA. 2012. URL: https://www.pdffiller.com/jsfiller-desk10/ ?projectId=6049b40aa44b1a50bc1261b8\&lp=true\#81c4f402f8bd4c0baed0c189ba1a5ec2 [accessed 2021-03-11]

5. Craike M, Bourke M, Hilland TA, Wiesner G, Pascoe MC, Bengoechea EG, et al. Correlates of physical activity among disadvantaged groups: a systematic review. Am J Prev Med 2019 Nov;57(5):700-715. [doi: 10.1016/j.amepre.2019.06.021] [Medline: 31630762 ]

6. Caspersen CJ, Powell KE, Christenson GM. Physical activity, exercise, and physical fitness: definitions and distinctions for health-related research. Public Health Rep 1985;100(2):126-131 [FREE Full text] [Medline: 3920711]

7. Gaskin CJ, Orellana L. Factors associated with physical activity and sedentary behavior in older adults from six low- and middle-income countries. Int J Environ Res Public Health 2018 May 03;15(5):908 [FREE Full text] [doi: 10.3390/ijerph15050908] [Medline: 29751561]

8. Su TT, Azzani M, Adewale AP, Thangiah N, Zainol R, Majid H. Physical activity and health-related quality of life among low-income adults in metropolitan Kuala Lumpur. J Epidemiol 2019;29(2):43-49. [doi: 10.2188/jea.je20170183]

9. Sawa R, Asai T, Doi T, Misu S, Murata S, Ono R. The association between physical activity, including physical activity intensity, and fear of falling differs by fear severity in older adults living in the community. J Gerontol B Psychol Sci Soc Sci 2020 Apr 16;75(5):953-960. [doi: 10.1093/geronb/gby103] [Medline: 30219902]

10. Falsarella G, Gasparotto LPR, Barcelos CC, Moretto MC, Pascoa MA, Ferreira TCBR, et al. Body composition as a frailty marker for the elderly community. Clin Interv Aging 2015 Oct:1661. [doi: 10.2147/cia.s84632]

11. Pirrie M, Saini G, Angeles R, Marzanek F, Parascandalo J, Agarwal G. Risk of falls and fear of falling in older adults residing in public housing in Ontario, Canada: findings from a multisite observational study. BMC Geriatr 2020 Jan 09;20(1):11 [FREE Full text] [doi: 10.1186/s12877-019-1399-1] [Medline: 31918674]

12. Thiamwong L, Sole ML, Ng BP, Welch GF, Huang HJ, Stout JR. Assessing fall risk appraisal through combined physiological and perceived fall risk measures using innovative technology. J Gerontol Nurs 2020 Apr 01;46(4):41-47. [doi: 10.3928/00989134-20200302-01] [Medline: 32219456]

13. Delbaere K, Close JCT, Brodaty H, Sachdev P, Lord SR. Determinants of disparities between perceived and physiological risk of falling among elderly people: cohort study. Br Med J 2010 Aug 19;341(aug18 4):4165. [doi: 10.1136/bmj.c4165]

14. Rodríguez-Molinero A, Gálvez-Barrón C, Narvaiza L, Miñarro A, Ruiz J, Valldosera E, et al. A two-question tool to assess the risk of repeated falls in the elderly. PLoS One 2017 May 10;12(5):- [FREE Full text] [doi: 10.1371/journal.pone.0176703] [Medline: 28489888 ]

15. Thiamwong L. A hybrid concept analysis of fall risk appraisal: integration of older adults' perspectives with an integrative literature review. Nurs Forum 2020 Apr 26;55(2):190-196. [doi: 10.1111/nuf.12415] [Medline: 31773750]

16. Gunn H, Cameron M, Hoang P, Lord S, Shaw S, Freeman J. Relationship between physiological and perceived fall risk in people with multiple sclerosis: implications for assessment and management. Arch Phys Med Rehabil 2018 Oct;99(10):2022-2029. [doi: 10.1016/j.apmr.2018.03.019] [Medline: 29698641]

17. $\mathrm{Ng} \mathrm{BP}$, Thiamwong L, He Q, Towne SD, Li Y. Discrepancies between perceived and physiological fall risks and repeated falls among community-dwelling medicare beneficiaries aged 65 years and older. Clin Gerontol 2020 Oct 22:1-13. [doi: 10.1080/07317115.2020.1833267] [Medline: 33090936]

18. Neri S, Gadelha AB, de David AC, Ferreira AP, Safons MP, Tiedemann A, et al. The association between body adiposity measures, postural balance, fear of falling, and fall risk in older community-dwelling women. J Geriatr Phys Ther 2019;42(3):94-100. [doi: 10.1519/JPT.0000000000000165] [Medline: 29227413]

19. Kim S, Leng XI, Kritchevsky SB. Body composition and physical function in older adults with various comorbidities. Innov Aging 2017 Mar 01;1(1):- [FREE Full text] [doi: 10.1093/geroni/igx008] [Medline: 30480107]

20. Janssen I, Heymsfield SB, Ross R. Low relative skeletal muscle mass (sarcopenia) in older persons is associated with functional impairment and physical disability. J Am Geriatr Soc 2002 May;50(5):889-896. [doi: 10.1046/j.1532-5415.2002.50216.x] [Medline: 12028177$]$

21. Chmelo E, Nicklas B, Davis C, Miller GD, Legault C, Messier S. Physical activity and physical function in older adults with knee osteoarthritis. J Phys Act Health 2013 Aug;10(6):777-783 [FREE Full text] [doi: 10.1123/jpah.10.6.777] [Medline: 23307503]

22. Cho B, Seo D, Lin H, Lohrmann DK, Chomistek AK. BMI and central obesity with falls among community-dwelling older adults. Am J Prev Med 2018 Apr;54(4):e59-e66. [doi: 10.1016/j.amepre.2017.12.020] [Medline: 29433954]

23. Tengvall M, Ellegård L, Malmros V, Bosaeus N, Lissner L, Bosaeus I. Body composition in the elderly: reference values and bioelectrical impedance spectroscopy to predict total body skeletal muscle mass. Clin Nutr 2009 Feb;28(1):52-58. [doi: 10.1016/j.clnu.2008.10.005] [Medline: 19010572]

24. Concept note: opening the GATE for assistive health technology: shifting the paradigm. World Health Organization. 2014. URL: https://www.who.int/phi/implementation/assistive_technology/concept_note.pdf [accessed 2021-03-12]

25. InBody S10. 2019. URL: https://inbodyusa.com/products/inbodys10/ [accessed 2021-03-12] 
26. BTrackS ${ }^{\mathrm{TM}}$ assess balance. Balance Tracking Systems. 2018. URL: https://balancetrackingsystems.com/assess-balance/ [accessed 2021-03-12]

27. Goble DJ. The BTrackSTM Balance Test is a valid predictor of older adults falling. 2018. URL: https:/ /balancetrackingsystems.com/wp-content/uploads/2019/05/Validating-BTrackS-FRA.pdf [accessed 2019-08-17]

28. Bassett DR, Troiano RP, McClain JJ, Wolff DL. Accelerometer-based physical activity: total volume per day and standardized measures. Med Sci Sports Exerc 2015 Apr;47(4):833-838. [doi: 10.1249/MSS.0000000000000468] [Medline: 25102292]

29. Poverty thresholds. United States Census Bureau. 2020. URL: https://www.census.gov/data/tables/time-series/demo/ income-poverty/historical-poverty-thresholds.html [accessed 2021-03-12]

30. Kuslansky G, Buschke H, Katz M, Sliwinski M, Lipton RB. Screening for Alzheimer's disease: the memory impairment screen versus the conventional three-word memory test. J Am Geriatr Soc 2002 Jun;50(6):1086-1091. [doi: 10.1046/j.1532-5415.2002.50265.x] [Medline: $\underline{12110070]}$

31. Böhm P, Peña-Casanova J, Gramunt N, Manero RM, Terrón C, Quiñones-Ubeda S. Spanish version of the Memory Impairment Screen (MIS): normative data and discriminant validity. Neurologia 2005 Oct;20(8):402-411. [Medline: 16217689]

32. Buschke H, Kuslansky G, Katz M, Stewart WF, Sliwinski MJ, Eckholdt HM, et al. Screening for dementia with the memory impairment screen. Neurology 1999 Jan 15;52(2):231-238. [doi: 10.1212/wnl.52.2.231] [Medline: 9932936]

33. Phelan E, Williams B, Meeker K, Bonn K, Frederick J, Logerfo J, et al. A study of the diagnostic accuracy of the PHQ-9 in primary care elderly. BMC Fam Pract 2010 Sep 01;11(1):63 [FREE Full text] [doi: 10.1186/1471-2296-11-63] [Medline: 20807445]

34. Familiar I, Ortiz-Panozo E, Hall B, Vieitez I, Romieu I, Lopez-Ridaura R, et al. Factor structure of the Spanish version of the Patient Health Questionnaire-9 in Mexican women. Int J Methods Psychiatr Res 2015 Mar 19;24(1):74-82 [FREE Full text] [doi: 10.1002/mpr.1461] [Medline: 25524806]

35. Yardley L, Beyer N, Hauer K, Kempen G, Piot-Ziegler C, Todd C. Development and initial validation of the Falls Efficacy Scale-International (FES-I). Age Ageing 2005 Nov;34(6):614-619. [doi: 10.1093/ageing/afi196] [Medline: 16267188]

36. Kempen GIJM, Yardley L, van Haastregt JCM, Zijlstra GAR, Beyer N, Hauer K, et al. The Short FES-I: a shortened version of the falls efficacy scale-international to assess fear of falling. Age Ageing 2008 Jan 11;37(1):45-50. [doi: 10.1093/ageing/afm157] [Medline: 18032400]

37. Delbaere K, Close JCT, Mikolaizak AS, Sachdev PS, Brodaty H, Lord SR. The Falls Efficacy Scale International (FES-I). A comprehensive longitudinal validation study. Age Ageing 2010 Mar 08;39(2):210-216. [doi: 10.1093/ageing/afp225] [Medline: 20061508]

38. del-Río-Valeiras M, Gayoso-Diz P, Santos-Pérez S, Rossi-Izquierdo M, Faraldo-García A, Vaamonde-Sánchez-Andrade I, et al. Is there a relationship between short FES-I test scores and objective assessment of balance in the older people with age-induced instability? Arch Gerontol Geriatr 2016 Jan;62:90-96. [doi: 10.1016/j.archger.2015.09.005] [Medline: 26412554]

39. Marques-Vieira CMA, Sousa LMM, Severino S, Sousa L, Caldeira S. Cross-cultural validation of the falls efficacy scale international in elderly: systematic literature review. J Clin Gerontol Geriatr 2016 Sep;7(3):72-76. [doi: 10.1016/j.jcgg.2015.12.002]

40. Bohannon RW, Crouch RPT. 1-Minute Sit-to-Stand Test: sytematic review of procedures, performance and clinimetric propeties. J Cardiopulm Rehabil Prev 2019;39(1):2-8. [doi: 10.1097/hcr.0000000000000336]

41. Jones CJ, Rikli RE, Beam WC. A 30-s chair-stand test as a measure of lower body strength in community-residing older adults. Res Q Exerc Sport 1999 Jun;70(2):113-119. [doi: 10.1080/02701367.1999.10608028] [Medline: 10380242]

42. Valentine JD, Simpson J, Worsfold C, Fisher K. A structural equation modelling approach to the complex path from postural stability to morale in elderly people with fear of falling. Disabil Rehabil 2011 Jun 09;33(4):352-359. [doi: 10.3109/09638288.2010.491575] [Medline: 20533873]

43. Goble DJ, Baweja HS. Postural sway normative data across the adult lifespan: results from 6280 individuals on the Balance Tracking System balance test. Geriatr Gerontol Int 2018 Aug 13;18(8):1225-1229. [doi: 10.1111/ggi.13452] [Medline: 29897159]

44. Levy SS, Thralls KJ, Kviatkovsky SA. Validity and reliability of a portable Balance Tracking System, BTrackS, in older adults. J Geriatr Phys Ther 2018;41(2):102-107. [doi: 10.1519/JPT.0000000000000111] [Medline: 27893566]

45. Buckinx F, Reginster J, Dardenne N, Croisiser J, Kaux J, Beaudart C, et al. Concordance between muscle mass assessed by bioelectrical impedance analysis and by dual energy X-ray absorptiometry: a cross-sectional study. BMC Musculoskelet Disord 2015 Mar 18;16(1):60 [FREE Full text] [doi: 10.1186/s12891-015-0510-9] [Medline: 25887598]

46. Gao KL, Tsang WW. Use of accelerometry to quantify the physical activity level of the elderly. Hong Kong Physiother J 2008;26(1):18-23. [doi: 10.1016/s1013-7025(09)70004-1]

47. Hornyak V, Brach JS, Wert DM, Hile E, Studenski S, VanSwearingen JM. What is the relation between fear of falling and physical activity in older adults? Arch Phys Med Rehabil 2013 Dec;94(12):2529-2534 [FREE Full text] [doi: 10.1016/j.apmr.2013.06.013] [Medline: 23816923]

48. Heesch KC, Hill RL, Aguilar-Farias N, van Uffelen JGZ, Pavey T. Validity of objective methods for measuring sedentary behaviour in older adults: a systematic review. Int J Behav Nutr Phys Act 2018 Nov 26;15(1):119 [FREE Full text] [doi: 10.1186/s12966-018-0749-2] [Medline: $\underline{\text { 30477509] }}$ 
49. Thiamwong L. Recruiting racial/ethnic community-dwelling older adults for a fall risk screening: a minority research scientist?s experience, in Southern Gerontological Society Conference. In: Proceedings of the SGS/GGS Joint Conference Moving Forward Together: Linking Research, Policy, and Practice. 2018 Presented at: SGS/GGS Joint Conference Moving Forward Together: Linking Research, Policy, and Practice; April 11-14, 2018; Legacy Lodge and Conference Center Lake Lanier, Georgia p. A URL: https://www.southerngerontologicalsociety.org/img/archives/2018/

2018SGS GGS-Program-Final-04-02.pdf

50. Centers for Disease Control and Prevention. Stopping elderly accidents, deaths, and injuries. STEDI-Older Adult Fall Prevention. 2017. URL: https://www.cdc.gov/steadi/ [accessed 2019-11-29]

51. Tudor-Locke C, Camhi S, Troiano R. A catalog of rules, variables, and definitions applied to accelerometer data in the National Health and Nutrition Examination Survey, 2003-2006. Prev Chronic Dis 2012 Jun;9:E113 [FREE Full text] [doi: 10.5888/pcd9.110332] [Medline: 22698174]

52. Trevisan C, Crippa A, Ek S, Welmer A, Sergi G, Maggi S, et al. Nutritional status, body mass index, and the risk of falls in community-dwelling older adults: a systematic review and meta-analysis. J Am Med Dir Assoc 2019 May;20(5):569-582. [doi: 10.1016/j.jamda.2018.10.027] [Medline: 30554987]

53. Palumbo P, Palmerini L, Bandinelli S, Chiari L. Fall risk assessment tools for elderly living in the community: can we do better? PLoS One 2015 Dec 30;10(12):- [FREE Full text] [doi: 10.1371/journal.pone.0146247] [Medline: 26716861]

54. Park S. Tools for assessing fall risk in the elderly: a systematic review and meta-analysis. Aging Clin Exp Res 2018 Jan 3;30(1):1-16. [doi: 10.1007/s40520-017-0749-0] [Medline: 28374345]

55. Perell KL, Nelson A, Goldman RL, Luther SL, Prieto-Lewis N, Rubenstein LZ. Fall risk assessment measures: an analytic review. J Gerontol A Biol Sci Med Sci 2001 Dec 01;56(12):M761-M766. [doi: 10.1093/gerona/56.12.m761] [Medline: $\underline{11723150]}$

56. Nunan S, Brown Wilson C, Henwood T, Parker D. Fall risk assessment tools for use among older adults in long-term care settings: a systematic review of the literature. Australas J Ageing 2018 Mar 23;37(1):23-33. [doi: 10.1111/ajag.12476] [Medline: 29168303]

57. McKechnie D, Pryor J, Fisher MJ. Predicting falls: considerations for screening tool selection vs. screening tool development. J Adv Nurs 2016 Sep 22;72(9):2238-2250. [doi: 10.1111/jan.12977] [Medline: 27101943]

58. Thiamwong L, Huang HJ, Ng BP, Yan X, Sole ML, Stout JR, et al. Shifting maladaptive fall risk appraisal in older adults through an in-home Physio-fEedback and Exercise pRogram (PEER): a pilot study. Clin Gerontol 2020 Nov 12;43(4):378-390. [doi: 10.1080/07317115.2019.1692120] [Medline: 31713464]

59. Thiamwong L, Stout JR, Sole ML, Ng BP, Yan X, Talbert S. Physio-Feedback and Exercise Program (PEER) improves balance, muscle strength, and fall risk in older adults. Res Gerontol Nurs 2020 Nov 01;13(6):289-296. [doi: 10.3928/19404921-20200324-01] [Medline: 32286669]

60. Marasinghe KM, Lapitan JM, Ross A. Assistive technologies for ageing populations in six low-income and middle-income countries: a systematic review. BMJ Innov 2015 Oct 11;1(4):182-195 [FREE Full text] [doi: 10.1136/bmjinnov-2015-000065] [Medline: 26688747]

61. Onken LS, Shoham V. Technology and the stage model of behavioral intervention development. In: Marsch L, Lord S, Dallery J, editors. Behavioral healthcare technology: using science-based innovations to transform practice. Oxford, United Kingdom: Oxford University Press; 2014:3-12.

62. Jo A, Coronel BD, Coakes CE, Mainous AG. Is there a benefit to patients using wearable devices such as Fitbit or health apps on mobiles? A systematic review. Am J Med 2019 Dec;132(12):1394-1400. [doi: 10.1016/j.amjmed.2019.06.018] [Medline: $\underline{31302077]}$

63. Valenzuela T, Okubo Y, Woodbury A, Lord SR, Delbaere K. Adherence to technology-based exercise programs in older adults: a systematic review. J Geriatr Phys Ther 2018;41(1):49-61. [doi: 10.1519/JPT.0000000000000095] [Medline: 27362526]
Abbreviations
AHT: assistive health technology
BBP: BTrackS balance plate
BBS: BTrackS balance system
BIA: bioelectrical impedance analysis
FES-I: Fall Efficacy Scale International
FOF: fear of falling
FRA: fall risk appraisal
ICC: intraclass correlation coefficient
PA: physical activity 
Edited by T Derrick;This paper was peer reviewed by the National Institutes of Health. Submitted 22.01.21; accepted 19.02.21; published 07.04.21.

Please cite as:

Thiamwong L, Stout JR, Park JH, Yan X

Technology-Based Fall Risk Assessments for Older Adults in Low-Income Settings: Protocol for a Cross-sectional Study JMIR Res Protoc 2021;10(4):e27381

URL: https://www.researchprotocols.org/2021/4/e27381

doi: $10.2196 / 27381$

PMID:

CLadda Thiamwong, Jeffrey R Stout, Joon-Hyuk Park, Xin Yan. Originally published in JMIR Research Protocols (http://www.researchprotocols.org), 07.04.2021. This is an open-access article distributed under the terms of the Creative Commons Attribution License (https://creativecommons.org/licenses/by/4.0/), which permits unrestricted use, distribution, and reproduction in any medium, provided the original work, first published in JMIR Research Protocols, is properly cited. The complete bibliographic information, a link to the original publication on http://www.researchprotocols.org, as well as this copyright and license information must be included. 